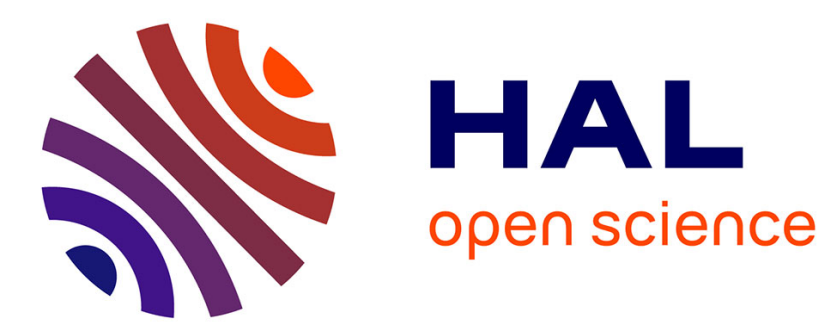

\title{
Numerical analysis of a finite volume scheme for a seawater intrusion model with cross-diffusion in an unconfined aquifer
}

\author{
Ahmed Ait Hammou Oulhaj
}

\section{To cite this version:}

Ahmed Ait Hammou Oulhaj. Numerical analysis of a finite volume scheme for a seawater intrusion model with cross-diffusion in an unconfined aquifer. Numerical Methods for Partial Differential Equations, 2018, 10.1002/num.22234 . hal-01432197

\section{HAL Id: hal-01432197 https://hal.science/hal-01432197}

Submitted on 18 Jun 2017

HAL is a multi-disciplinary open access archive for the deposit and dissemination of scientific research documents, whether they are published or not. The documents may come from teaching and research institutions in France or abroad, or from public or private research centers.
L'archive ouverte pluridisciplinaire HAL, est destinée au dépôt et à la diffusion de documents scientifiques de niveau recherche, publiés ou non, émanant des établissements d'enseignement et de recherche français ou étrangers, des laboratoires publics ou privés. 


\title{
NUMERICAL ANALYSIS OF A FINITE VOLUME SCHEME FOR A SEAWATER INTRUSION MODEL WITH CROSS-DIFFUSION IN AN UNCONFINED AQUIFER
}

\author{
AHMED AIT HAMMOU OULHAJ
}

\begin{abstract}
We consider a degenerate parabolic system modeling the flow of fresh and saltwater in a porous medium in the context of seawater intrusion. We propose and analyze a finite volume scheme based on two-point flux approximation with upwind mobilities. The scheme preserves at the discrete level the main features of the continuous problem, namely the nonnegativity of the solutions, the decay of the energy and the control of the entropy and its dissipation. Based on these nonlinear stability results, we show that the scheme converges towards a weak solution to the problem. Numerical results are provided to illustrate the behavior of the model and of the scheme.
\end{abstract}

Keywords. Unsaturated porous media flow, seawater intrusion, nonlinear discretization, entropy stability, convergence analysis, cross-diffusion, unconfined aquifer

AMS subjects classification. 65M12, 65M08, 76S05

\section{INTRODUCTION}

1.1. Presentation of the continuous problem. We are interested in the study of seawater intrusion problem in coastal regions. If they are densely populated areas, the intensive extraction of freshwater yields to local water table depression and saltwater from the sea can enter the ground and replace the freshwater. This causes sea intrusion problem. In these zones, the optimal exploitation of freshwater and the limitation of seawater intrusion are a challenge for the future. Since freshwater and saltwater are miscible fluids, we have a transition zone separating them caused by hydrodynamic dispersion. In the literature, there exists several modelling approaches. The first approach is to assume that the fluids are immiscible and the domains occupied by each fluid are separated by an interface called sharp interface. It is obtained by vertical integration based on the assumptions that no mass transfer occurs between the fresh and the saltwater and by the so-called Dupuit approximation. Physically, this approach is not totally correct but enables to follow the saltwater front. We refer to $[7,8,9,38,37,3]$ for more details about this first approach. The second approach consists in considering the existence of a transition zone with variable concentrations of salt. It is difficult to tackle this approach from theorical and numerical points of view (see $[13,35,36])$. The third approach is to assume that the fluids are miscible, and no interface between these fluids. This modelling approach is physically correct, but it has the disadvantage that it is impossible to follow explicitly the interface (see [14]). The fourth approach is a mixed approach (sharp-diffuse). Recently in [16] the authors derive this model for seawater intrusion phenomena in unconfined aquifer. It combines the efficiency 
of the sharp interface approach with the physical realism of the diffuse interface one. For mathematical analysis of sharp-diffuse interfaces see [15].

In this paper, we consider the first approach, by focusing on the seawater intrusion model in an unconfined aquifer, obtained in [28] considering the formal asymptotic limit as the aspect ratio between the thickness and the horizontal length of the porous medium tends to zero. In our setting $\xi$ is a nonnegative function expressing the height of the interface between the saltwater and the freshwater while $h \geq \xi$ is the height of the interface separating the freshwater and the dry soil. We assume that the bottom of the porous medium, which is located at $b \neq 0$, is impermeable, cf. Figure 1. Moreover we assume quasi-horizontal displacements (Dupuit approximation), hence we get a $2 \mathrm{D}$-vertically averaged model. This assumption is reasonable since the thickness of the aquifer is small compared to the horizontal length of the aquifer.

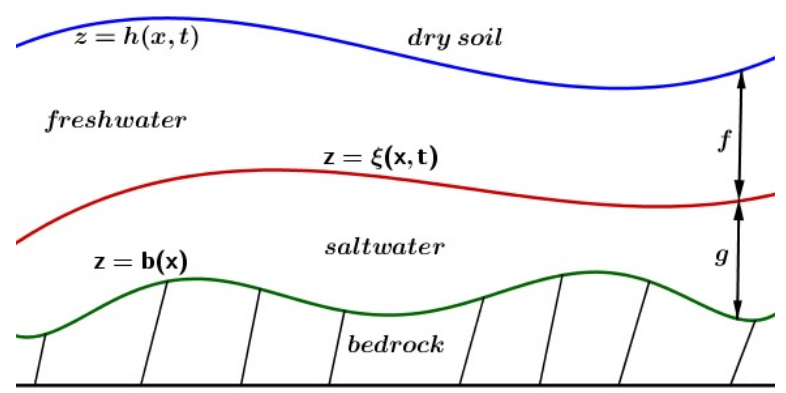

FiguRE 1. Description of an unconfined aquifer

The evolution of $\xi$ and $h$ is given by the following cross-diffusion system of a degenerate parabolic equation

$$
\left\{\begin{array}{lll}
\partial_{t}(h-\xi)-\nabla \cdot\left((h-\xi) \nabla\left(\left(1-\varepsilon_{0}\right) h\right)\right) & =0 & \text { in } \quad \Omega \times(0, T)=: \Omega_{T} \\
\partial_{t} \xi-\nabla \cdot\left((\xi-b) \nabla\left(\left(1-\varepsilon_{0}\right) h+\varepsilon_{0} \xi\right)\right) & =0 & \text { in } \quad \Omega \times(0, T),
\end{array}\right.
$$

with $\Omega \subset \mathbb{R}^{2}$ is a polygonal open bounded subset, and $T>0$ a finite time horizon. The parameter $\varepsilon_{0}$ is given by

$$
\varepsilon_{0}=\frac{\rho_{s}-\rho_{f}}{\rho_{s}},
$$

where $\rho_{s}$ (resp. $\rho_{f}$ ) is the mass density of the fluid saltwater (resp. freshwater) (assumed to be constant with $0<\rho_{f}<\rho_{s}$ ). We set $f=h-\xi, g=\xi-b$ and

$$
\nu=1-\varepsilon_{0}=\frac{\rho_{f}}{\rho_{s}} \in(0,1) .
$$

The system (1) then rewrites:

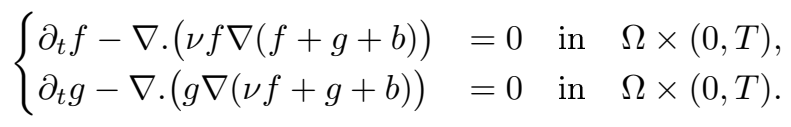

It is supplemented with no-flux boundary conditions

$$
\nabla f \cdot \mathbf{n}=\nabla g \cdot \mathbf{n}=0, \quad \text { on } \partial \Omega \times(0, T),
$$


where $\mathbf{n}$ is the unit normal to the boundary $\partial \Omega$, and initial data

$$
f_{\mid t=0}=f_{0}, \quad g_{\mid t=0}=g_{0},
$$

with $f_{0}, g_{0} \in L^{\infty}(\Omega)$ and

$$
f_{0}, g_{0} \geq 0, \quad \text { a.e } x \in \Omega \text {. }
$$

Before describing more precisely our results, let us mention that the problems of kind (3), have been the object of several studies. In particular the Muskat problem for thin fluid layers, which models (see [20]) the movement of two fluids with densities and viscosities in a porous medium in one dimension. The authors in $[20,21]$ studied the classical solutions of such problem. Moreover, weak solutions are established under different assumptions in [19, 29, 30].

We recall (see $[19,29,30])$ the definition of entropy functional:

$$
\mathfrak{H}(f, g)=\int_{\Omega}\left[\Gamma(g)+\frac{1}{\nu} \Gamma(f)\right] \mathrm{d} \mathbf{x}, \quad \text { where } \Gamma(s)=s \log s-s+1,
$$

and of the energy functional:

$$
\mathfrak{E}(f, g)=\int_{\Omega}\left[\frac{\nu}{2}(f+g+b)^{2}+\frac{1-\nu}{2}(g+b)^{2}\right] \mathrm{d} \mathbf{x} .
$$

Multiplying (formally) the first equation of (3) by $\frac{1}{\nu} \log f$ and the second equation by $\log g$, integrating over $\Omega$ and summing both equations, yields the classical entropy/dissipation property:

$$
\frac{\mathrm{d}}{\mathrm{d} t} \mathfrak{H}(f, g)+\frac{1-\nu}{2} \int_{\Omega}\left[(\nabla f)^{2}+(\nabla g)^{2}\right] \mathrm{d} \mathbf{x} \leq \frac{1}{2(\nu+1)} \int_{\Omega}(\nabla b)^{2} \mathrm{~d} \mathbf{x} .
$$

Moreover multiplying (formally) the first equation of (3) by $\nu(f+g+b)$ and the second equation by $\nu f+g+b$, integrating over $\Omega$ and summing both equations, yields that the energy functional decreases along time:

$$
\frac{\mathrm{d}}{\mathrm{d} t} \mathfrak{E}(f, g)+\int_{\Omega}\left[\nu^{2} f(\nabla(f+g+b))^{2}+g(\nabla(\nu f+g+b))^{2}\right] \mathrm{d} \mathbf{x}=0 .
$$

Let us mention that the cross-diffusion systems are extensively presented in different domain as ecology, biology, chemistry, and others. In [26] the author propose and analyze a finite volume scheme for the Patlak-Keller-Segel (PKS) chemotaxis model. In [10] the authors studie the PKS model with additional cross diffusion. We refer to [5] for the analysis of a finite volume method for a cross diffusion model in population dynamics. See [34, 32] for the numerical analysis for a seawater intrusion problem in a unconfined aquifer with finite element method approximation. In [1] the authors propose a finite element method and a finite volume method, and compare the results given by these two methods. In [17] the authors address the question of global existence for the sharp interface approach. For an analysis of a finite volume scheme for two-phase immiscible flow in porous media, used in petroleum engineering, we can refer to these papers [33, 25].

In this work, we propose a finite volume scheme for the problem (3). This scheme is based on a two-point flux approximation with upwind mobilities. It is designed in order to preserve at the discrete level the main features of the continuous problem: the nonnegativity of the solutions, the decay of the energy (8), and the control of the entropy and its dissipation (7). 
1.2. The numerical scheme. In this section, we explicit the discretization of the problem (3)-(4) we will study in this paper. The time discretization relies on backward Euler scheme, while the space discretization relies on a finite volume approach (see e.g [23]), with two-point flux approximation and upstream mobility.

Let $\Omega \subset \mathbb{R}^{2}$ be an open, bounded, polygonal subset. An admissible mesh of $\Omega$ is given by a family $\mathcal{T}$ of a control volumes (open and convex polygons), a family $\mathcal{E}$ of edges, and a family of points $\left(x_{K}\right)_{K \in \mathcal{T}}$ which satisfy Definition 9.1 in [23]. This definition implies that the straight line between two neighboring centers of cells $\left(x_{K}, x_{L}\right)$ is orthogonal to the edge $\sigma=K \mid L$.

We distinguish the interior edges $\sigma \in \mathcal{E}_{\text {int }}$ and the boundary edges $\sigma \in \mathcal{E}_{\text {ext }}$. The set of edges $\mathcal{E}$ equals the union $\mathcal{E}_{\text {int }} \cup \mathcal{E}_{\text {ext }}$. For a control volume $K \in \mathcal{T}$, we denote by $\mathcal{E}_{K}$ the set of its edges, by $\mathcal{E}_{K \text {,int }}$ the set of its interior edges, and by $\mathcal{E}_{K \text {,ext }}$ the set of edges of $K$ included in $\partial \Omega$.

Furthermore, we denote by $d$ the distance in $\mathbb{R}^{2}$ and by $m$ the Lebesgue measure in $\mathbb{R}^{2}$ or $\mathbb{R}$. We assume that the family of meshes satisfies the following regularity requirement: there exists $\zeta>0$ such that for all $K \in \mathcal{T}$ and all $\sigma \in \mathcal{E}_{\text {int }, K}$ with $\sigma=K \mid L$, it holds

$$
d\left(x_{K}, \sigma\right) \geq \zeta d\left(x_{K}, x_{L}\right)
$$

For all $\sigma \in \mathcal{E}_{\text {int }, K}$ with $\sigma=K \mid L$, we define $d_{\sigma}=d\left(x_{K}, x_{L}\right)$, and the transmissibility coefficient

$$
\tau_{\sigma}=\frac{m(\sigma)}{d_{\sigma}}, \quad \sigma \in \mathcal{E}
$$

The size of the mesh is defined by

$$
\delta=\max _{K \in \mathcal{T}}(\operatorname{diam}(K)) .
$$

In order to avoid heavier notations, we restrict our study to the case of a uniform time discretization of $(0, T)$. However, all the results presented in this paper can be extended to general time discretizations without any technical difficulty. In what follows, we assume that the spatial mesh is fixed and does not change with the time step. Let $T>0$ be some final time and $M_{T}$ the number of time steps. Then the time step size and the time points are given by, respectively,

$$
\Delta t=\frac{T}{M_{T}}, \quad t^{n}=n \Delta t, \quad 0 \leq n \leq M_{T} .
$$

We denote by $\mathcal{D}$ an admissible space-time discretization of $\Omega_{T}=\Omega \times(0, T)$ composed of an admissible mesh $\mathcal{T}$ of $\Omega$ and the values $\Delta t$ and $M_{T}$. The size of this space-time discretization $\mathcal{D}$ is defined by $\eta=\max (\delta, \Delta t)$.

The initial conditions are discretized by

$$
\begin{aligned}
& f_{\mathcal{T}}^{0}=\sum_{K \in \mathcal{T}} f_{K}^{0} \mathbf{1}_{K}, \quad \text { where } f_{K}^{0}=\frac{1}{m(K)} \int_{K} f_{0}(x) \mathrm{d} x, \quad \forall K \in \mathcal{T}, \\
& g_{\mathcal{T}}^{0}=\sum_{K \in \mathcal{T}} g_{K}^{0} \mathbf{1}_{K}, \quad \text { where } g_{K}^{0}=\frac{1}{m(K)} \int_{K} g_{0}(x) \mathrm{d} x, \quad \forall K \in \mathcal{T},
\end{aligned}
$$

and $\mathbf{1}_{K}$ is the characteristic function on $K$. Denoting by $f_{K}^{n}$ and $g_{K}^{n}$ approximations of the mean value of $f\left(., t^{n}\right)$ and $g\left(., t^{n}\right)$ on $K$, respectively. Taking for $b_{K}$ the value of $b$ in a fixed point of $K$ (for instance, the center of gravity of $K$ ), where $b$ is a 
regular function, and assume that $b_{K} \geq 0 \quad \forall K \in \mathcal{T}$. The discretization of problem (3) is given by the following set of nonlinear equations:

$$
\begin{aligned}
& m(K) \frac{f_{K}^{n+1}-f_{K}^{n}}{\Delta t} \\
& \quad+\sum_{\sigma \in \mathcal{E}_{\mathrm{int}, K}} \tau_{\sigma} f_{\sigma}^{n+1} \nu\left(\left(f_{K}^{n+1}-f_{L}^{n+1}\right)+\left(g_{K}^{n+1}-g_{L}^{n+1}\right)+\left(b_{K}-b_{L}\right)\right)=0,
\end{aligned}
$$

and

$$
\begin{aligned}
& m(K) \frac{g_{K}^{n+1}-g_{K}^{n}}{\Delta t} \\
& \quad+\sum_{\sigma \in \mathcal{E}_{\text {int }, K}} \tau_{\sigma} g_{\sigma}^{n+1}\left(\nu\left(f_{K}^{n+1}-f_{L}^{n+1}\right)+\left(g_{K}^{n+1}-g_{L}^{n+1}\right)+\left(b_{K}-b_{L}\right)\right)=0,
\end{aligned}
$$

for $K \in \mathcal{T}$ and $0 \leq n \leq M_{T}-1$, where

$$
f_{\sigma}^{n+1}= \begin{cases}\left(f_{K}^{n+1}\right)^{+} & \text {if }\left(f_{K}^{n+1}-f_{L}^{n+1}\right)+\left(g_{K}^{n+1}-g_{L}^{n+1}\right)+\left(b_{K}-b_{L}\right) \geq 0 \\ \left(f_{L}^{n+1}\right)^{+} & \text {if }\left(f_{K}^{n+1}-f_{L}^{n+1}\right)+\left(g_{K}^{n+1}-g_{L}^{n+1}\right)+\left(b_{K}-b_{L}\right)<0\end{cases}
$$

and

$$
g_{\sigma}^{n+1}= \begin{cases}\left(g_{K}^{n+1}\right)^{+} & \text {if } \nu\left(f_{K}^{n+1}-f_{L}^{n+1}\right)+\left(g_{K}^{n+1}-g_{L}^{n+1}\right)+\left(b_{K}-b_{L}\right) \geq 0 \\ \left(g_{L}^{n+1}\right)^{+} & \text {if } \nu\left(f_{K}^{n+1}-f_{L}^{n+1}\right)+\left(g_{K}^{n+1}-g_{L}^{n+1}\right)+\left(b_{K}-b_{L}\right)<0\end{cases}
$$

where $x^{+}=\max (0, x)$. We next define the numerical approximation $\left(f_{\mathcal{D}}, g_{\mathcal{D}}\right)$ of $(f, g)$ by

$$
\begin{aligned}
& f_{\mathcal{D}}(x, t)=\sum_{\substack{K \in \mathcal{T} \\
0 \leq n \leq M_{T}-1}} f_{K}^{n+1} \mathbf{1}_{K \times\left(t^{n}, t^{n+1}\right]}(x, t), \text { and } \\
& g_{\mathcal{D}}(x, t)=\sum_{\substack{K \in \mathcal{T} \\
0 \leq n \leq M_{T}-1}} g_{K}^{n+1} \mathbf{1}_{K \times\left(t^{n}, t^{n+1}\right]}(x, t) .
\end{aligned}
$$

We also define approximations of the gradients $\nabla^{\mathcal{D}} f_{\mathcal{D}}$ and $\nabla^{\mathcal{D}} g_{\mathcal{D}}$ of $f$ and $g$, respectively. To this end, we introduce that: for $K \in \mathcal{T}$

- If $\sigma=K \mid L \in \mathcal{E}_{\mathrm{int}, K}, \mathfrak{D}_{K, L}$ is the cell ("diamond") whose vertices are given by $x_{K}, x_{L}$, and the end points of the edge $\sigma=K \mid L$.

- $\mathfrak{D}_{K, \sigma}=\mathfrak{D}_{K, L} \cap K$ is the cell ("triangle") whose vertices are given by $x_{K}$ and the end points of the edge $\sigma=K \mid L$.

The approximate gradient $\nabla^{\mathcal{D}} S_{\mathcal{D}}$ (with $S=f$, or $S=g$ ) is a piecewise constant function, defined in $\Omega_{T}$ by

$$
\nabla^{\mathcal{D}} S_{\mathcal{D}}(x, t)=-\frac{m(\sigma)}{m\left(\mathfrak{D}_{K, L}\right)}\left(S_{K}^{n+1}-S_{L}^{n+1}\right) \nu_{K, L}, \quad x \in \mathfrak{D}_{K, L}, t \in\left(t^{n}, t^{n+1}\right],
$$

where $\nu_{K, L}$ is the unit vector normal to $\sigma$ and outward to $K$.

1.3. Main results and outline of the paper. The scheme (13)-(16) amounts to a nonlinear system to be solved at each time step. The existence of a solution to this system is therefore non trivial. The first result we highlight is thus the existence of a nonnegative solution to the scheme (13)-(16), the stability in terms of the discrete entropy, and the decay of the discrete energy. 
Theorem 1.1. There exists (at least) one solution $\left(f_{K}^{n+1}, g_{K}^{n+1}\right)_{K \in \mathcal{T}, n \in\left\{0, \ldots, M_{T}-1\right\}}$ to the scheme (13)-(16). Moreover, $f_{K}^{n} \geq 0, g_{K}^{n} \geq 0$ for all $K \in \mathcal{T}$ and for all $n \in\left\{0, \ldots, M_{T}\right\}$, and there exists $C$ depending only on $\Omega, f_{0}, g_{0}, \nu$ and $b$ such that

$$
\begin{array}{r}
\sup _{n \in\left\{0, \ldots, M_{T}-1\right\}} \mathfrak{H}^{n+1}+\sum_{n=0}^{M_{T}-1} \Delta t \sum_{\substack{\sigma \in \mathcal{E}_{\text {int }} \\
\sigma=K \mid L}} \tau_{\sigma}\left[\left(f_{K}^{n+1}-f_{L}^{n+1}\right)^{2}+\left(g_{K}^{n+1}-g_{L}^{n+1}\right)^{2}\right] \\
\leq C(1+T),
\end{array}
$$

and

$$
\begin{aligned}
& \sup _{n \in\left\{0, \ldots, M_{T}-1\right\}} \mathfrak{E}^{n+1} \\
& +\sum_{n=0}^{M_{T}-1} \Delta t \sum_{\substack{\sigma \in \mathcal{E}_{i n t} \\
\sigma=K \mid L}} \tau_{\sigma} f_{\sigma}^{n+1} \nu^{2}\left(\left(f_{K}^{n+1}-f_{L}^{n+1}\right)+\left(g_{K}^{n+1}-g_{L}^{n+1}\right)+\left(b_{K}-b_{L}\right)\right)^{2} \\
& +\sum_{n=0}^{M_{T}-1} \Delta t \sum_{\substack{\sigma \in \mathcal{E}_{i n t} \\
\sigma=K \mid L}} \tau_{\sigma} g_{\sigma}^{n+1}\left(\nu\left(f_{K}^{n+1}-f_{L}^{n+1}\right)+\left(g_{K}^{n+1}-g_{L}^{n+1}\right)+\left(b_{K}-b_{L}\right)\right)^{2} \leq C .
\end{aligned}
$$

Our second result concerns the convergence of the scheme to a weak solution of (3)-(4). Let $\left(\mathcal{D}_{m}\right)_{m>0}$ be a family of admissible space-time discretization of $\Omega_{T}$. We denote by $\left(\mathcal{T}_{m}\right)_{m>0}$ the corresponding meshes of $\Omega$, with $\operatorname{size}\left(\mathcal{T}_{m}\right)=\delta_{m} \longrightarrow 0$, as $m \rightarrow 0$. We define $\left(f_{m}, g_{m}\right):=\left(f_{\mathcal{D}_{m}}, g_{\mathcal{D}_{m}}\right)$ the sequence of approximate solutions constructed on the discretization $\mathcal{D}_{m}$. We set $\nabla^{m}:=\nabla^{\mathcal{D}_{m}}$.

Theorem 1.2. Let $\left(\mathcal{D}_{m}\right)_{m>0}$ be a sequence of admissible discretizations satisfying (9) uniformly in $m$, and $\lim _{m \rightarrow+\infty} \eta_{m}=0$. Let $\left(f_{m}, g_{m}\right)$ be a sequence of finite volume solutions to (13)-(16). Then there exists $(f, g)$ such that, up a subsequence,

$$
\begin{aligned}
& f_{m} \longrightarrow f \quad \text { in } L^{r}\left(\Omega_{T}\right), \forall r<4, \text { and } \nabla^{m} f_{m} \longrightarrow \nabla f \quad \text { weakly in } L^{2}\left(\Omega_{T}\right)^{2} \text {, } \\
& g_{m} \longrightarrow g \quad \text { in } L^{r}\left(\Omega_{T}\right), \forall r<4 \text {, and } \nabla^{m} g_{m} \longrightarrow \nabla g \text { weakly in } L^{2}\left(\Omega_{T}\right)^{2} \text {, }
\end{aligned}
$$

and $(f, g) \in L^{2}\left(0, T ; H^{1}(\Omega)\right)^{2}$ is a weak solution to (3)-(4) in the following sense

$$
\begin{aligned}
& \int_{0}^{T} \int_{\Omega}\left(f \partial_{t} \psi-\nu f \nabla(f+g+b) \cdot \nabla \psi\right) \mathrm{d} x \mathrm{~d} t+\int_{\Omega} f_{0} \psi(., 0) \mathrm{d} x=0 \\
& \int_{0}^{T} \int_{\Omega}\left(g \partial_{t} \psi-g \nabla(\nu f+g+b) \cdot \nabla \psi\right) \mathrm{d} x \mathrm{~d} t+\int_{\Omega} g_{0} \psi(., 0) \mathrm{d} x=0
\end{aligned}
$$

for all test functions $\psi \in C_{0}^{\infty}(\Omega \times[0, T))$.

The paper is organized as follows. The existence of nonnegative solution is shown in Section 2. Discrete counterparts of the entropy/entropy-dissipation (7) and energy/energy-dissipation (8) relations are established in Section 3. Section 4 is devoted to the convergence proof of the scheme. This proof is based first on the compactness of the sequence of approximate solutions and then on the identification of the limit. We finally present numerical experiments in Section 5, to illustrate the behaviour of the model and of the scheme. 


\section{Existence of A NONNEGATIVE DisCRETE SOLUTIONS}

First all, we prove the positivity of the discrete solutions. This estimate allows to prove the existence of a solution to the nonlinear system (13)-(16).

Proposition 2.1. For all $K \in \mathcal{T}, n \geq 0$,

$$
f_{K}^{n} \geq 0, \quad g_{K}^{n} \geq 0
$$

hence

$$
\sum_{K \in \mathcal{T}} m(K) f_{K}^{n}=\sum_{K \in \mathcal{T}} m(K) f_{K}^{0}=\left\|f_{0}\right\|_{L^{1}(\Omega)}
$$

and

$$
\sum_{K \in \mathcal{T}} m(K) g_{K}^{n}=\sum_{K \in \mathcal{T}} m(K) g_{K}^{0}=\left\|g_{0}\right\|_{L^{1}(\Omega)}
$$

Proof. The property (20) clearly holds for $n=0$ thanks to (6). Assume now that (20) holds at time step $n$, and assume that

$$
f_{K}^{n+1}<0, \quad \text { for some } K \in \mathcal{T} .
$$

In view of the definition (15) of $f_{\sigma}^{n+1}$ one has that

$$
\begin{aligned}
f_{K}^{n+1}= & -\frac{\nu \Delta t}{m(K)} \sum_{\sigma \in \mathcal{E}_{\mathrm{int}, K}} \tau_{\sigma}(\underbrace{\left(f_{K}^{n+1}\right)^{+}}_{=0}\left[\left(f_{K}^{n+1}-f_{L}^{n+1}\right)+\left(g_{K}^{n+1}-g_{L}^{n+1}\right)+\left(b_{K}-b_{L}\right)\right]^{+} \\
& \left.-\left(f_{L}^{n+1}\right)^{+}\left[\left(f_{K}^{n+1}-f_{L}^{n+1}\right)+\left(g_{K}^{n+1}-g_{L}^{n+1}\right)+\left(b_{K}-b_{L}\right)\right]^{-}\right)+f_{K}^{n} \geq 0,
\end{aligned}
$$

yielding a contradiction, ensuring that

$$
f_{K}^{n+1} \geq 0, \quad \forall K \in \mathcal{T}, \forall n \geq 0 .
$$

Proving that $g_{K}^{n} \geq 0$ for all $K \in \mathcal{T}, \forall n \geq 0$, is similar.

We will prove the existence of a solution, we follow the methodology proposed in [22], using a topological degree argument [18, 31].

Proposition 2.2. Let $\mathcal{D}$ be an admissible discretization of $\Omega \times(0, T)$. There exists (at least) one solution to the scheme (13)-(16).

Proof. Let $\mu \in[0,1]$, and define $\left(f_{K, \mu}^{n+1}, g_{K, \mu}^{n+1}\right)_{K}$ as the solution of the scheme: $\forall K \in$ $\mathcal{T}$

$$
\begin{aligned}
& m(K) \frac{f_{K, \mu}^{n+1}-f_{K}^{n}}{\Delta t} \\
& \quad+\mu \sum_{\sigma \in \mathcal{E}_{\mathrm{int}, K}} \tau_{\sigma} f_{\sigma, \mu}^{n+1} \nu\left(\left(f_{K, \mu}^{n+1}-f_{L, \mu}^{n+1}\right)+\left(g_{K, \mu}^{n+1}-g_{L, \mu}^{n+1}\right)+\left(b_{K}-b_{L}\right)\right)=0, \\
& m(K) \frac{g_{K, \mu}^{n+1}-g_{K}^{n}}{\Delta t} \\
& \quad+\mu \sum_{\sigma \in \mathcal{E}_{\mathrm{int}, K}} \tau_{\sigma} g_{\sigma, \mu}^{n+1}\left(\nu\left(f_{K, \mu}^{n+1}-f_{L, \mu}^{n+1}\right)+\left(g_{K, \mu}^{n+1}-g_{L, \mu}^{n+1}\right)+\left(b_{K}-b_{L}\right)\right)=0 .
\end{aligned}
$$


Reproducing the proof of Proposition 2.1, one can show that

$$
f_{K, \mu}^{n+1} \geq 0, \text { and } g_{K, \mu}^{n+1} \geq 0 \quad \forall \mu \in[0,1],
$$

hence

$$
\sum_{K \in \mathcal{T}} m(K) f_{K, \mu}^{n+1}=\sum_{K \in \mathcal{T}} m(K) f_{K}^{0}=\left\|f_{0}\right\|_{L^{1}(\Omega)},
$$

and

$$
\sum_{K \in \mathcal{T}} m(K) g_{K, \mu}^{n+1}=\sum_{K \in \mathcal{T}} m(K) g_{K}^{0}=\left\|g_{0}\right\|_{L^{1}(\Omega)} .
$$

Therefore, for all $K \in \mathcal{T}$, one has

$$
0 \leq f_{K, \mu}^{n+1} \leq \frac{\left\|f_{0}\right\|_{L^{1}(\Omega)}}{m(K)} \leq \frac{\left\|f_{0}\right\|_{L^{1}(\Omega)}}{\min _{K \in \mathcal{T}} m(K)}:=m_{f},
$$

and

$$
0 \leq g_{K, \mu}^{n+1} \leq \frac{\left\|g_{0}\right\|_{L^{1}(\Omega)}}{m(K)} \leq \frac{\left\|g_{0}\right\|_{L^{1}(\Omega)}}{\min _{K \in \mathcal{T}} m(K)}:=m_{g} .
$$

Define the compact subset $\mathcal{K}=\left[-1, m_{f}+1\right]^{\# \mathcal{T}} \times\left[-1, m_{g}+1\right]^{\# \mathcal{T}}$ of $\mathbb{R}^{\# \mathcal{T}} \times \mathbb{R}^{\# \mathcal{T}}$, and define the function $\mathcal{H}\left(\left(f_{K}, g_{K}\right)_{K}, \mu\right): \mathbb{R}^{\# \mathcal{T}} \times \mathbb{R}^{\# \mathcal{T}} \times[0,1] \rightarrow \mathbb{R}^{\# \mathcal{T}} \times \mathbb{R}^{\# \mathcal{T}}$ by: $\forall K \in \mathcal{T}$,

$$
\begin{aligned}
& \mathcal{H}\left(\left(f_{K}, g_{K}\right)_{K}, \mu\right)=\left(m(K) \frac{f_{K, \mu}^{n+1}-f_{K}^{n}}{\Delta t}\right. \\
&+\mu \sum_{\sigma \in \mathcal{E}_{\mathrm{int}, K}} \tau_{\sigma} f_{\sigma, \mu}^{n+1} \nu\left(\left(f_{K, \mu}^{n+1}-f_{L, \mu}^{n+1}\right)+\left(g_{K, \mu}^{n+1}-g_{L, \mu}^{n+1}\right)+\left(b_{K}-b_{L}\right)\right), \\
&\left.m(K) \frac{g_{K, \mu}^{n+1}-g_{K}^{n}}{\Delta t}+\mu \sum_{\sigma \in \mathcal{E}_{\mathrm{int}, K}} \tau_{\sigma} g_{\sigma, \mu}^{n+1}\left(\nu\left(f_{K, \mu}^{n+1}-f_{L, \mu}^{n+1}\right)+\left(g_{K, \mu}^{n+1}-g_{L, \mu}^{n+1}\right)+\left(b_{K}-b_{L}\right)\right)\right) .
\end{aligned}
$$

The function $\mathcal{H}$ is uniformly continuous on $\mathcal{K} \times[0,1]$, and it follows from (23) that for all $\mu \in[0,1]$, the nonlinear system

$$
\mathcal{H}\left(\left(f_{K}, g_{K}\right)_{K}, \mu\right)=(0,0),
$$

cannot admit any solution on $\partial \mathcal{K}$. Therefore, the corresponding topological degree $\delta(\mathcal{H}, \mathcal{K})(\mu)$ is constant w.r.t $\mu$. For $\mu=0$ the linear system $\mathcal{H}\left(\left(f_{K}, g_{K}\right)_{K}, 0\right)$ admits a unique solution, and the topological degree is equal to 1 . Hence, the nonlinear system (25) admits at least one solution for $\mu=1$, ensuring the existence of a solution to the scheme (13)-(16).

\section{ENTROPy AND ENERGY ESTIMATES}

The goal of this section is to establish discrete counterparts to the entropy/entropydissipation estimate (7) and energy/energy-dissipation estimate (8). In what follows, $\left(f_{K}^{n}, g_{K}^{n}\right)$ denotes a solution to the scheme (13)-(16). The proof of Theorem 1.1 is based on suitable estimates, which are shown below. This section also contains some results that will be useful in the convergence proof of Section 4 . 
3.1. Discrete $L^{2}\left(0, T ; H^{1}(\Omega)\right)$ semi-norm. We first have to define the space $\mathcal{X}(\mathcal{D})$ the solution belongs to, and the discrete $L^{2}\left(0, T ; H^{1}(\Omega)\right)$ semi-norm.

Definition 3.1. We denote by $\mathcal{X}(\mathcal{D})$ the functional space:

$$
\mathcal{X}(\mathcal{D})=\left\{\begin{array}{c}
u \in L^{\infty}\left(\Omega_{T}\right) / u \text { is constant on } K \times\left(t^{n}, t^{n+1}\right] \\
\forall K \in \mathcal{T}, \forall n \in\left\{0, \cdots, M_{T}-1\right\}
\end{array}\right\} .
$$

Definition 3.2. ( Discrete $L^{2}\left(0, T ; H^{1}(\Omega)\right)$ semi-norm ) We define the discrete $L^{2}\left(0, T ; H^{1}(\Omega)\right)$ semi-norm on $\mathcal{X}(\mathcal{D})$ by:

$$
|u|_{1, \mathcal{D}}=\left(\sum_{n=0}^{M_{T}-1} \Delta t \sum_{\substack{\sigma \in \mathcal{E}_{i n t} \\ \sigma=K \mid L}} \tau_{\sigma}\left(u_{K}^{n+1}-u_{L}^{n+1}\right)^{2}\right)^{1 / 2} .
$$

Remark 3.3. Note that

$$
\left\|\nabla^{\mathcal{D}} u\right\|_{L^{2}\left(\Omega_{T}\right)}=\sqrt{2}|u|_{1, \mathcal{D}} .
$$

3.2. Entropy estimate. We introduce a discrete version of entropy functional:

$$
\mathfrak{H}^{n}:=\mathfrak{H}\left(f_{K}^{n}, g_{K}^{n}\right)=\sum_{K \in \mathcal{T}} m(K)\left(\frac{1}{\nu} \Gamma\left(f_{K}^{n}\right)+\Gamma\left(g_{K}^{n}\right)\right) .
$$

Proposition 3.4. (Entropy stability) For all $n \in\left\{0, \ldots, M_{T}-1\right\}$, one has

$$
\begin{aligned}
& \mathfrak{H}^{n+1}-\mathfrak{H}^{n}+\frac{1-\nu}{2} \Delta t \sum_{\substack{\sigma \in \mathcal{E}_{i n t} \\
\sigma=K \mid L}} \tau_{\sigma}\left[\left(f_{K}^{n+1}-f_{L}^{n+1}\right)^{2}+\left(g_{K}^{n+1}-g_{L}^{n+1}\right)^{2}\right] \\
& \leq \frac{\Delta t}{2(\nu+1)} \sum_{\substack{\sigma \in \mathcal{E}_{i n t} \\
\sigma=K \mid L}} \tau_{\sigma}\left(b_{K}-b_{L}\right)^{2} .
\end{aligned}
$$

Proof. We multiply (13) by $\Delta t \frac{\log f_{K}^{n+1}}{\nu}$ and summing over $K \in \mathcal{T}$, and (14) by $\Delta t \log g_{K}^{n+1}$ and summing over $K \in \mathcal{T}$, provides that:

$$
A+B+C=0
$$

where

$$
\begin{aligned}
& A=\sum_{K \in \mathcal{T}} m(K)\left[\frac{1}{\nu}\left(f_{K}^{n+1}-f_{K}^{n}\right) \log f_{K}^{n+1}+\left(g_{K}^{n+1}-g_{K}^{n}\right) \log g_{K}^{n+1}\right], \\
& B=\Delta t \sum_{K \in \mathcal{T}} \sum_{\sigma \in \mathcal{E}_{\mathrm{int}, K}} \tau_{\sigma} f_{\sigma}^{n+1}\left(\left(f_{K}^{n+1}-f_{L}^{n+1}\right)+\left(g_{K}^{n+1}-g_{L}^{n+1}\right)+\left(b_{K}-b_{L}\right)\right) \log f_{K}^{n+1}, \\
& C=\Delta t \sum_{K \in \mathcal{T}} \sum_{\sigma \in \mathcal{E}_{\mathrm{int}, K}} \tau_{\sigma} g_{\sigma}^{n+1}\left(\nu\left(f_{K}^{n+1}-f_{L}^{n+1}\right)+\left(g_{K}^{n+1}-g_{L}^{n+1}\right)+\left(b_{K}-b_{L}\right)\right) \log g_{K}^{n+1} .
\end{aligned}
$$

By the convexity of $\Gamma$, we find that

$$
\mathfrak{H}^{n+1}-\mathfrak{H}^{n}=\sum_{K \in \mathcal{T}} m(K)\left[\frac{1}{\nu}\left(\Gamma\left(f_{K}^{n+1}\right)-\Gamma\left(f_{K}^{n}\right)\right)+\Gamma\left(g_{K}^{n+1}\right)-\Gamma\left(g_{K}^{n}\right)\right] \leq A .
$$


We can rewrite $B$ and $C$ as:

$$
\begin{gathered}
B=\Delta t \sum_{\substack{\sigma \in \mathcal{E}_{\text {int }} \\
\sigma=K \mid L}} \tau_{\sigma} f_{\sigma}^{n+1}\left(\left(f_{K}^{n+1}-f_{L}^{n+1}\right)+\left(g_{K}^{n+1}-g_{L}^{n+1}\right)+\right. \\
\left(\log f_{K}^{n+1}-\log f_{L}^{n+1}\right), \\
C=\Delta t \sum_{\substack{\sigma \in \mathcal{E}_{\text {int }} \\
\sigma=K \mid L}} \tau_{\sigma} g_{\sigma}^{n+1}\left(\nu\left(f_{K}^{n+1}-f_{L}^{n+1}\right)+\left(g_{K}^{n+1}-g_{L}^{n+1}\right)+\left(b_{K}-b_{L}\right)\right) \times \\
\left(\log g_{K}^{n+1}-\log g_{L}^{n+1}\right) .
\end{gathered}
$$

It follows from the convexity of exp that

$$
a(\log a-\log b) \geq a-b \geq b(\log a-\log b) \quad \forall a, b \in[0,+\infty[,
$$

where we have used the convention $\log (0)=-\infty$ and $0 \log (0)=0$. Hence, in view of the definition (15) and (16) of the upwind mobilities, one has

$$
\begin{array}{r}
B \geq \Delta t \sum_{\substack{\sigma \in \mathcal{E}_{\text {int }} \\
\sigma=K \mid L}} \tau_{\sigma}\left(\left(f_{K}^{n+1}-f_{L}^{n+1}\right)^{2}+\left(g_{K}^{n+1}-g_{L}^{n+1}\right)\left(f_{K}^{n+1}-f_{L}^{n+1}\right)\right. \\
\left.+\left(b_{K}-b_{L}\right)\left(f_{K}^{n+1}-f_{K}^{n+1}\right)\right), \\
C \geq \Delta t \sum_{\substack{\sigma \in \mathcal{E}_{\text {int }} \\
\sigma=K \mid L}} \tau_{\sigma}\left(\left(g_{K}^{n+1}-g_{L}^{n+1}\right)^{2}+\nu\left(g_{K}^{n+1}-g_{L}^{n+1}\right)\left(f_{K}^{n+1}-f_{L}^{n+1}\right)\right. \\
\left.+\left(b_{K}-b_{L}\right)\left(g_{K}^{n+1}-g_{L}^{n+1}\right)\right) .
\end{array}
$$

Combining these inequalities, one deduces that

$$
\begin{aligned}
\mathfrak{H}^{n+1}-\mathfrak{H}^{n}+\Delta t \sum_{\substack{\sigma \in \mathcal{E}_{\text {int }} \\
\sigma=K \mid L}} \tau_{\sigma}\left(f_{K}^{n+1}-f_{L}^{n+1}\right)^{2}+\Delta t \sum_{\substack{\sigma \in \mathcal{E}_{\text {int }} \\
\sigma=K \mid L}} \tau_{\sigma}\left(g_{K}^{n+1}-g_{L}^{n+1}\right)^{2} \\
+(\nu+1) \Delta t \sum_{\substack{\sigma \in \mathcal{E}_{\text {int }} \\
\sigma=K \mid L}} \tau_{\sigma}\left(f_{K}^{n+1}-f_{L}^{n+1}\right)\left(g_{K}^{n+1}-g_{L}^{n+1}\right) \leq D,
\end{aligned}
$$

where

$$
D=-\Delta t \sum_{\substack{\sigma \in \mathcal{E}_{\text {int }} \\ \sigma=K \mid L}} \tau_{\sigma}\left(b_{K}-b_{L}\right)\left[\left(f_{K}^{n+1}-f_{L}^{n+1}\right)+\left(g_{K}^{n+1}-g_{L}^{n+1}\right)\right] .
$$

Using the Young inequality, one has

$$
D \leq \frac{1}{2 \epsilon} \Delta t \sum_{\substack{\sigma \in \mathcal{E}_{\text {int }} \\ \sigma=K \mid L}} \tau_{\sigma}\left(b_{K}-b_{L}\right)^{2}+\frac{\epsilon}{2} \Delta t \sum_{\substack{\sigma \in \mathcal{E}_{\text {int }} \\ \sigma=K \mid L}} \tau_{\sigma}\left[\left(f_{K}^{n+1}-f_{L}^{n+1}\right)+\left(g_{K}^{n+1}-g_{L}^{n+1}\right)\right]^{2},
$$


for all $\epsilon>0$. We choose $\epsilon=1+\nu$, we have

$$
\begin{gathered}
D \leq \frac{1}{2(\nu+1)} \Delta t \sum_{\substack{\sigma \in \mathcal{E}_{\text {int }} \\
\sigma=K \mid L}} \tau_{\sigma}\left(b_{K}-b_{L}\right)^{2} \\
+\frac{\nu+1}{2} \Delta t \sum_{\substack{\sigma \in \mathcal{E}_{\text {int }} \\
\sigma=K \mid L}} \tau_{\sigma}\left[\left(f_{K}^{n+1}-f_{L}^{n+1}\right)^{2}+\left(g_{K}^{n+1}-g_{L}^{n+1}\right)^{2}\right] \\
+(\nu+1) \Delta t \sum_{\substack{\sigma \in \mathcal{E}_{\text {int }} \\
\sigma=K \mid L}} \tau_{\sigma}\left[\left(f_{K}^{n+1}-f_{L}^{n+1}\right)\left(g_{K}^{n+1}-g_{L}^{n+1}\right)\right] .
\end{gathered}
$$

Finally, one has

$$
\begin{aligned}
\mathfrak{H}^{n+1}-\mathfrak{H}^{n}+\frac{1-\nu}{2} \Delta t \sum_{\substack{\sigma \in \mathcal{E}_{\text {int }} \\
\sigma=K \mid L}} \tau_{\sigma}\left(f_{K}^{n+1}-f_{L}^{n+1}\right)^{2} \\
+\frac{1-\nu}{2} \Delta t \sum_{\substack{\sigma \in \mathcal{E}_{\text {int }} \\
\sigma=K \mid L}} \tau_{\sigma}\left(g_{K}^{n+1}-g_{L}^{n+1}\right)^{2} \leq \frac{1}{2(\nu+1)} \Delta t \sum_{\substack{\sigma \in \mathcal{E}_{\text {int }} \\
\sigma=K \mid L}} \tau_{\sigma}\left(b_{K}-b_{L}\right)^{2},
\end{aligned}
$$

where $1-\nu>0$ thanks to $(2)$.

Corollary 3.5. There exists $C_{1}$ depending only on $T, \Omega, f_{0}, g_{0}, \nu$ and $b$ such that

$$
\mathfrak{H}^{M_{T}}+\frac{1-\nu}{2}\left(\left|f_{\mathcal{D}}\right|_{1, \mathcal{D}}^{2}+\left|g_{\mathcal{D}}\right|_{1, \mathcal{D}}^{2}\right) \leq C_{1}
$$

Proof. Summing (27) over $n=0, \ldots, M_{T}-1$ provides

$$
\begin{aligned}
\mathfrak{H}^{M_{T}}+\frac{1-\nu}{2} \sum_{n=0}^{M_{T}-1} \Delta t \sum_{\substack{\sigma \in \mathcal{E}_{\text {int }} \\
\sigma=K \mid L}} \tau_{\sigma}\left[\left(f_{K}^{n+1}-f_{L}^{n+1}\right)^{2}+\left(g_{K}^{n+1}-g_{L}^{n+1}\right)^{2}\right] \\
\leq \mathfrak{H}^{0}+\frac{1}{2(\nu+1)} \sum_{n=0}^{M_{T}-1} \Delta t \sum_{\substack{\sigma \in \mathcal{E}_{\text {int }} \\
\sigma=K \mid L}} \tau_{\sigma}\left(b_{K}-b_{L}\right)^{2} .
\end{aligned}
$$

As a consequence of Jensen's inequality, one has

$$
\mathfrak{H}^{0}=\int_{\Omega}\left[\frac{1}{\nu} \Gamma\left(f_{K}^{0}\right)+\Gamma\left(g_{K}^{0}\right)\right] \mathrm{d} x \leq \int_{\Omega}\left[\frac{1}{\nu} \Gamma\left(f_{0}\right)+\Gamma\left(g_{0}\right)\right] \mathrm{d} x<+\infty,
$$

and

$$
\sum_{n=0}^{M_{T}-1} \Delta t \sum_{\substack{\sigma \in \mathcal{E}_{\text {int }} \\ \sigma=K \mid L}} \tau_{\sigma}\left(b_{K}-b_{L}\right)^{2} \leq T|\Omega|\|\nabla b\|_{\infty}^{2}
$$

concluding the proof of Corollary 3.5.

We obtain immediately thanks to (26), the following discrete $L^{2}\left(\Omega_{T}\right)$ estimate on the discrete gradients:

$$
\left\|\nabla^{\mathcal{D}} f_{\mathcal{D}}\right\|_{L^{2}\left(\Omega_{T}\right)}^{2}+\left\|\nabla^{\mathcal{D}} g_{\mathcal{D}}\right\|_{L^{2}\left(\Omega_{T}\right)}^{2} \leq 2 C_{1} .
$$


3.3. Energy estimate. The current subsection is devoted to the proof of the discrete energy estimate. We introduce a discrete version of energy functional:

$$
\mathfrak{E}^{n}:=\mathfrak{E}\left(f_{K}^{n}, g_{K}^{n}\right)=\sum_{K \in \mathcal{T}} m(K)\left(\frac{\nu}{2}\left(f_{K}^{n}+g_{K}^{n}+b_{K}\right)^{2}+\frac{1-\nu}{2}\left(g_{K}^{n}+b_{K}\right)^{2}\right) .
$$

Proposition 3.6. For all $n \in\left\{0, \ldots, M_{T}-1\right\}$, one has

$$
\begin{aligned}
& \mathfrak{E}^{n+1}+\Delta t \sum_{\substack{\sigma \in \mathcal{E}_{\text {int }} \\
\sigma=K \mid L}} \tau_{\sigma} f_{\sigma}^{n+1} \nu^{2}\left(\left(f_{K}^{n+1}-f_{L}^{n+1}\right)+\left(g_{K}^{n+1}-g_{L}^{n+1}\right)+\left(b_{K}-b_{L}\right)\right)^{2} \\
& +\Delta t \sum_{\substack{\sigma \in \mathcal{E}_{\text {int }} \\
\sigma=K \mid L}} \tau_{\sigma} g_{\sigma}^{n+1}\left(\nu\left(f_{K}^{n+1}-f_{L}^{n+1}\right)+\left(g_{K}^{n+1}-g_{L}^{n+1}\right)+\left(b_{K}-b_{L}\right)\right)^{2} \leq \mathfrak{E}^{n} .
\end{aligned}
$$

Proof. We multiply (13) (resp. (14)) by $\Delta t \nu\left(f_{K}^{n+1}+g_{K}^{n+1}+b_{K}\right)\left(\right.$ resp. $\Delta t\left(\nu f_{K}^{n+1}+\right.$ $\left.\left.g_{K}^{n+1}+b_{K}\right)\right)$ and sum over $K \in \mathcal{T}$. Summing both equalities and reorganizing the sums, we get $A+B=0$, where

$$
\begin{gathered}
A=\sum_{K \in \mathcal{T}} m(K)\left[\nu\left(f_{K}^{n+1}-f_{K}^{n}\right)\left(f_{K}^{n+1}+g_{K}^{n+1}+b_{K}\right)\right] \\
\quad+\sum_{K \in \mathcal{T}} m(K)\left[\left(g_{K}^{n+1}-g_{K}^{n}\right)\left(\nu f_{K}^{n+1}+g_{K}^{n+1}+b_{K}\right)\right], \\
B=\Delta t \sum_{\substack{\sigma \in \mathcal{E}_{\text {int }} \\
\sigma=K \mid L}} \tau_{\sigma} f_{\sigma}^{n+1} \nu^{2}\left(\left(f_{K}^{n+1}-f_{L}^{n+1}\right)+\left(g_{K}^{n+1}-g_{L}^{n+1}\right)+\left(b_{K}-b_{L}\right)\right)^{2} \\
+\Delta t \sum_{\substack{\sigma \in \mathcal{E}_{\text {int }} \\
\sigma=K \mid L}} \tau_{\sigma} g_{\sigma}^{n+1}\left(\nu\left(f_{K}^{n+1}-f_{L}^{n+1}\right)+\left(g_{K}^{n+1}-g_{L}^{n+1}\right)+\left(b_{K}-b_{L}\right)\right)^{2} .
\end{gathered}
$$

One has

$$
\begin{aligned}
A=\sum_{K \in \mathcal{T}} m(K) & {\left[\nu\left(\left(f_{K}^{n+1}+g_{K}^{n+1}+b_{K}\right)-\left(f_{K}^{n}+g_{K}^{n}+b_{K}\right)\right)\left(f_{K}^{n+1}+g_{K}^{n+1}+b_{K}\right)\right] } \\
+ & \sum_{K \in \mathcal{T}} m(K)\left[(1-\nu)\left(\left(g_{K}^{n+1}+b_{K}\right)-\left(g_{K}^{n}+b_{K}\right)\right)\left(g_{K}^{n+1}+b_{K}\right)\right] .
\end{aligned}
$$

We use the following inequality: $(a-b) a \geq \frac{1}{2}\left(a^{2}-b^{2}\right), \forall a, b \in \mathbb{R}$, to get

$$
\begin{aligned}
A \geq \sum_{K \in \mathcal{T}} m(K) & {\left[\frac{\nu}{2}\left(\left(f_{K}^{n+1}+g_{K}^{n+1}+b_{K}\right)^{2}-\left(f_{K}^{n}+g_{K}^{n}+b_{K}\right)^{2}\right)\right] } \\
+ & \sum_{K \in \mathcal{T}} m(K)\left[\frac{1-\nu}{2}\left(\left(g_{K}^{n+1}+b_{K}\right)^{2}-\left(g_{K}^{n}+b_{K}\right)^{2}\right)\right]=\mathfrak{E}^{n+1}-\mathfrak{E}^{n} .
\end{aligned}
$$

Remark 3.7. In the discrete counterpart (30) of (8), the equality is remplaced by an inequality. But as well as in the continuous setting, the function $\mathfrak{E}$ decreases along time. 
Corollary 3.8. There exists $C_{2}$ depending only on $f_{0}, g_{0}, b, \nu$ and $\Omega$ such that

$$
\left\|f_{\mathcal{D}}\right\|_{L^{\infty}\left(0, T ; L^{2}(\Omega)\right)}+\left\|g_{\mathcal{D}}\right\|_{L^{\infty}\left(0, T ; L^{2}(\Omega)\right)} \leq C_{2} .
$$

Proof. Summing (30) over $n=0, \ldots, M_{T}-1$, we obtain immediately thanks the positivity of $f_{\sigma}^{n+1}$ and $g_{\sigma}^{n+1}$ that

$$
\mathfrak{E}^{n} \leq \mathfrak{E}^{0} .
$$

Using the Cauchy-Schwarz inequality, we obtain

$$
\mathfrak{E}^{0} \leq \sum_{K \in \mathcal{T}} m(K)\left(\frac{\nu+\nu^{2}}{2}\left(f_{K}^{0}\right)^{2}+2\left(g_{K}^{0}\right)^{2}+2 b_{K}^{2}\right) .
$$

Moreover, for $s=f$, or $g$ one has

$$
\sum_{K \in \mathcal{T}} m(K)\left(s_{K}^{0}\right)^{2} \leq\left\|s_{0}\right\|_{L^{2}(\Omega)}^{2}, \quad \text { and } \quad \sum_{K \in \mathcal{T}} m(K) b_{K}^{2} \leq|\Omega|\|b\|_{\infty}^{2} .
$$

Hence

$$
\mathfrak{E}^{0} \leq\left\|f_{0}\right\|_{L^{2}(\Omega)}^{2}+2\left\|g_{0}\right\|_{L^{2}(\Omega)}^{2}+2|\Omega|\|b\|_{\infty}^{2}<+\infty
$$

On the other hand since $b_{K}, f_{K}$ and $g_{K}$ are nonnegative for all $K \in \mathcal{T}$, then we have

We deduce that

$$
\mathfrak{E}^{n} \geq \sum_{K \in \mathcal{T}} m(K) \frac{\nu}{2}\left[\left(f_{K}^{n}\right)^{2}+\left(g_{K}^{n}\right)^{2}\right] .
$$

$$
\sum_{K \in \mathcal{T}} m(K)\left[\left(f_{K}^{n}\right)^{2}+\left(g_{K}^{n}\right)^{2}\right] \leq \frac{2}{\nu} \mathfrak{E}^{n} \leq \frac{2}{\nu} \mathfrak{E}^{0},
$$

concluding the proof of Corollary 3.8 .

Then we deduce from Proposition 3.6 that

Corollary 3.9. There exists $C_{3}$ depending only on $f_{0}, g_{0}, b, \nu$ and $\Omega$ such that

$$
\begin{aligned}
& \sum_{n=0}^{M_{T}-1} \Delta t \sum_{\substack{\sigma \in \mathcal{E}_{\text {int }} \\
\sigma=K \mid L}} \tau_{\sigma} f_{\sigma}^{n+1} \nu^{2}\left(\left(f_{K}^{n+1}-f_{L}^{n+1}\right)+\left(g_{K}^{n+1}-g_{L}^{n+1}\right)+\left(b_{K}-b_{L}\right)\right)^{2}+ \\
& \sum_{n=0}^{M_{T}-1} \Delta t \sum_{\substack{\sigma \in \mathcal{E}_{\text {int }} \\
\sigma=K \mid L}} \tau_{\sigma} g_{\sigma}^{n+1}\left(\nu\left(f_{K}^{n+1}-f_{L}^{n+1}\right)+\left(g_{K}^{n+1}-g_{L}^{n+1}\right)+\left(b_{K}-b_{L}\right)\right)^{2} \leq C_{3} .
\end{aligned}
$$

\section{Convergence analysis}

This section is devoted to the compactness of the approximate solution. Our goal is to show the strong compactness of the sequences $\left(f_{m}\right)_{m>0}$ and $\left(g_{m}\right)_{m>0}$ in $L^{2}\left(\Omega_{T}\right)$ and the weak compactness in $L^{2}\left(\Omega_{T}\right)^{2}$ of the approximate gradient of $f_{m}$ and $g_{m}$ defined by (17). As a first step, we show in $\S 4.1$ the appropriate compactness properties on the reconstructed discrete solutions. Then we identify in $\S 4.2$ the limit value (whose existence is ensured thanks to the compactness properties) as the weak solution to the problem (3). 
4.1. Compactness properties of discrete solutions. As it is classical for unsteady problems, we need to prove some time-compactness for the approximate solutions. We make use of the time-compactness result for degenerate parabolic equations proposed in [6], as an alternative to the classical technique that consists in estimating the time-translates (see [4] in the continuous setting and [23] in the discrete setting).

Lemma 4.1. There exists $C_{4}$ depending only on $\zeta, T, f_{0}, g_{0}, \nu$ and $b$ such that

$$
\begin{aligned}
& \text { (31) } \sum_{n=0}^{M_{T}-1} \sum_{K \in \mathcal{T}} m(K)\left(f_{K}^{n+1}-f_{K}^{n}\right) \varphi\left(x_{K}, t_{n+1}\right) \leq C_{4}\|\nabla \varphi\|_{L^{\infty}\left(\Omega_{T}\right)}, \quad \varphi \in \mathcal{C}_{c}^{\infty}\left(\Omega_{T}\right) . \\
& \text { (32) } \sum_{n=0}^{M_{T}-1} \sum_{K \in \mathcal{T}} m(K)\left(g_{K}^{n+1}-g_{K}^{n}\right) \varphi\left(x_{K}, t_{n+1}\right) \leq C_{4}\|\nabla \varphi\|_{L^{\infty}\left(\Omega_{T}\right)}, \quad \varphi \in \mathcal{C}_{c}^{\infty}\left(\Omega_{T}\right) .
\end{aligned}
$$

Proof. For the sake of readability, we denote by $\varphi_{K}^{n+1}=\varphi\left(x_{K}, t_{n+1}\right)$ for all $K \in \mathcal{T}$ and all $n \in\left\{0, \ldots, M_{T}-1\right\}$. We multiply the scheme (13) by $\Delta t \varphi_{K}^{n+1}$ and sum for $K \in \mathcal{T}$, for $n \in\left\{0, \ldots, M_{T}-1\right\}$. This yields:

$$
A=B,
$$

where

$$
A=\sum_{n=0}^{N} \sum_{K \in \mathcal{T}} m(K)\left(f_{K}^{n+1}-f_{K}^{n}\right) \varphi_{K}^{n+1}
$$

and

$$
\begin{aligned}
B=-\nu \sum_{n=0}^{M_{T}-1} \Delta t \sum_{\substack{\sigma \in \mathcal{E}_{\text {int }} \\
\sigma=K \mid L}} \tau_{\sigma} f_{\sigma}^{n+1}\left[\left(f_{K}^{n+1}-f_{L}^{n+1}\right)+\right. & \left(g_{K}^{n+1}-g_{L}^{n+1}\right) \\
& \left.+\left(b_{K}-b_{L}\right)\right]\left(\varphi_{K}^{n+1}-\varphi_{L}^{n+1}\right) .
\end{aligned}
$$

Using the Cauchy-Schwarz inequality, we get

$$
\begin{aligned}
|B|^{2} & \leq \sum_{n=0}^{M_{T}-1} \Delta t \sum_{\substack{\sigma \in \mathcal{E}_{\text {int }} \\
\sigma=K \mid L}} \tau_{\sigma} f_{\sigma}^{n+1}\left(\varphi_{K}^{n+1}-\varphi_{L}^{n+1}\right)^{2} \\
& \times \sum_{n=0}^{M_{T}-1} \Delta t \sum_{\substack{\sigma \in \mathcal{E}_{\text {int }} \\
\sigma=K \mid L}} \tau_{\sigma} f_{\sigma}^{n+1} \nu^{2}\left[\left(f_{K}^{n+1}-f_{L}^{n+1}\right)+\left(g_{K}^{n+1}-g_{L}^{n+1}\right)+\left(b_{K}-b_{L}\right)\right]^{2} .
\end{aligned}
$$

Moreover $f_{\sigma}^{n+1} \in\left[\min \left(f_{K}^{n+1}, f_{L}^{n+1}\right), \max \left(f_{K}^{n+1}, f_{L}^{n+1}\right)\right]$, hence

$$
0 \leq f_{\sigma}^{n+1} \leq f_{K}^{n+1}+f_{L}^{n+1}, \quad \forall \sigma \in \mathcal{E}, \forall n \in\left\{0, \ldots, M_{T}-1\right\} .
$$

Using Corollary 3.9 , we get

$$
|B|^{2} \leq C_{3} \sum_{n=0}^{M_{T}-1} \Delta t \sum_{\substack{\sigma \in \mathcal{E}_{\text {int }} \\ \sigma=K \mid L}} \tau_{\sigma}\left(f_{K}^{n+1}+f_{L}^{n+1}\right) d\left(x_{K}, x_{L}\right)^{2}\|\nabla \varphi\|_{L^{\infty}\left(\Omega_{T}\right)}^{2} .
$$


Observe that in two space dimensions,

$$
\sum_{K \in \mathcal{T}} \sum_{\sigma \in \mathcal{E}_{\text {int }, K}} d_{\sigma} m(\sigma) \leq 2 \sum_{K \in \mathcal{T}} m(K)
$$

By (10), (9) one has

$$
\begin{aligned}
& \sum_{n=0}^{M_{T}-1} \Delta t \sum_{\substack{\sigma \in \mathcal{E}_{\text {int }} \\
\sigma=K \mid L}} \tau_{\sigma}\left(f_{K}^{n+1}+f_{L}^{n+1}\right) d\left(x_{K}, x_{L}\right)^{2} \\
& \leq \sum_{n=0}^{M_{T}-1} \Delta t \sum_{\substack{\sigma \in \mathcal{E}_{\text {int }} \\
\sigma=K \mid L}} m(\sigma) d\left(x_{K}, x_{L}\right)\left(f_{K}^{n+1}+f_{L}^{n+1}\right) \\
& \leq \sum_{n=0}^{M_{T}-1} \Delta t \sum_{K \in \mathcal{T}} f_{K}^{n+1} \sum_{\sigma \in \mathcal{E}_{\text {int }, K}} m(\sigma) d\left(x_{K}, x_{L}\right) \\
& \quad \leq \frac{2 T}{\zeta} \sum_{K \in \mathcal{T}} m(K) f_{K}^{n+1} \leq \frac{2 T}{\zeta}\left\|f_{0}\right\|_{L^{1}(\Omega)},
\end{aligned}
$$

thanks the mass conservation (21). Hence

$$
\sum_{n=0}^{M_{T}-1} \Delta t \sum_{\substack{\sigma \in \mathcal{E}_{\text {int }} \\ \sigma=K \mid L}} m(\sigma) d\left(x_{K}, x_{L}\right) f_{\sigma}^{n+1} \leq \frac{2 T}{\zeta}\left\|f_{0}\right\|_{L^{1}(\Omega)} .
$$

This concludes the proof of (31). The proof of (32) is similar.

We can apply the [6, Theorem 3.9], we conclude that

Proposition 4.2. Let $\left(\mathcal{D}_{m}\right)_{m>1}$ be a family of admissible space-time discretization of $\Omega_{T}$ such that (9) holds. Let $\left(f_{m}\right)_{m}$ and $\left(g_{m}\right)_{m}$ be the corresponding sequence of discrete solution to the scheme (13)-(16), then up to an unlabeled subsequence, there exits $f \in L^{2}\left(0, T ; H^{1}(\Omega)\right)$ and $g \in L^{2}\left(0, T ; H^{1}(\Omega)\right)$ such that

$$
f_{m} \underset{m \rightarrow+\infty}{\longrightarrow} f \text {, a.e in } \Omega_{T} \text {, and } g_{m} \underset{m \rightarrow+\infty}{\longrightarrow} g \text {, a.e in } \Omega_{T} \text {. }
$$

$\left(f_{m}\right)_{m>0}$ and $\left(g_{m}\right)_{m>0}$ are uniformly bounded in $L^{\infty}\left(0 ; T, L^{2}(\Omega)\right)$ thanks to Corollary 3.8. By Corollary 3.5 and Sobolev embedding, $\left(f_{m}\right)_{m>0}$ and $\left(g_{m}\right)_{m>0}$ are bounded in $L^{2}\left(0, T ; L^{p}(\Omega)\right)$, with $p<+\infty$. Then, thanks to Riez-Thorin theorem, $\left(f_{m}\right)_{m>0}$ and $\left(g_{m}\right)_{m>0}$ are bounded in $L^{r}\left(\Omega_{T}\right)$ with $2<r<4$. Hence $\left(f_{m}\right)_{m>0}$ and $\left(g_{m}\right)_{m>0}$ are equi-integrable in $L^{r}\left(\Omega_{T}\right)$. Applying the Vitali's convergence theorem we deduce that

Lemma 4.3. Keeping the assumption and notations of Proposition 4.2, one has

$$
f_{m} \underset{m \rightarrow+\infty}{\longrightarrow} f, \quad \text { strongly in } L^{r}\left(\Omega_{T}\right), \quad \text { for all } r<4 \text {, }
$$

and

$$
g_{m} \underset{m \rightarrow+\infty}{\longrightarrow} g, \quad \text { strongly in } L^{r}\left(\Omega_{T}\right), \quad \text { for all } r<4 \text {. }
$$

We show now the weak compactness in $L^{2}\left(\Omega_{T}\right)^{2}$ of the approximate gradient of $f_{m}$ and $g_{m}$ defined in (17): 
Proposition 4.4. Keeping the assumption and notations of Proposition 4.2, one has

$\nabla^{m} f_{m} \underset{m \rightarrow+\infty}{\longrightarrow} \nabla f$ weakly in $L^{2}\left(\Omega_{T}\right)^{2}$, and $\nabla^{m} g_{m} \underset{m \rightarrow+\infty}{\longrightarrow} \nabla g$ weakly in $L^{2}\left(\Omega_{T}\right)^{2}$.

Proof. Thanks to (29) $\nabla^{\mathcal{D}} f_{\mathcal{D}}$ and $\nabla^{\mathcal{D}} g_{\mathcal{D}}$ are bounded in $L^{2}\left(\Omega_{T}\right)^{2}$, then there exists a subsequence of $\nabla^{\mathcal{D}} f_{\mathcal{D}}$ and of $\nabla^{\mathcal{D}} g_{\mathcal{D}}$ (still labeled $\nabla^{\mathcal{D}} f_{D}$ and $\nabla^{\mathcal{D}} g_{\mathcal{D}}$ ) and two function $\Theta, \Xi \in L^{2}\left(\Omega_{T}\right)^{2}$ such that

$$
\nabla^{m} f_{m} \underset{m \rightarrow+\infty}{\longrightarrow} \Theta, \text { weakly in } L^{2}\left(\Omega_{T}\right)^{2},
$$

and

$$
\nabla^{m} g_{m} \underset{m \rightarrow+\infty}{\longrightarrow} \Xi, \quad \text { weakly in } L^{2}\left(\Omega_{T}\right)^{2} .
$$

We refer to $[12,24]$ to prove that $\Theta=\nabla f$ and $\Xi=\nabla g$.

\subsection{Identification as a weak solution.}

Proposition 4.5. Let $(f, g)$ be as in Proposition 4.2, then $f$ and $g$ are the weak solution to (3)-(4) in the sense of (18) and (19).

Proof. Let $\psi \in C_{0}^{\infty}(\bar{\Omega} \times[0, T))$ be a test function and $\psi_{K}^{n+1}=\psi\left(x_{K}, t_{n+1}\right)$ for all $K \in \mathcal{T}$ and $n \in\left\{0, \ldots, M_{T}-1\right\}$. We first establish (18) from (13), and to obtain (19) from (14) is similar. In order to prove that $f$ is a weak solution, we multiply (13) by $\Delta t_{m} \psi_{K}^{n}$ and sum over $n \in\left\{0, \ldots, M_{T}-1\right\}$ and $K \in \mathcal{T}$, we obtain

$$
A_{m}+B_{m}=0,
$$

where

$$
\begin{aligned}
A_{m} & =\sum_{n=0}^{M_{T}-1} \sum_{K \in \mathcal{T}} m(K)\left(f_{K}^{n+1}-f_{L}^{n+1}\right) \psi_{K}^{n}, \\
B_{m} & =\nu \sum_{n=0}^{M_{T}-1} \Delta t_{m} \sum_{K \in \mathcal{T}} \psi_{K}^{n} \sum_{\sigma \in \mathcal{E}_{\text {int }, K}} \tau_{\sigma} f_{\sigma}^{n+1}\left[\left(f_{K}^{n+1}-f_{L}^{n+1}\right)+\left(g_{K}^{n+1}-g_{L}^{n+1}\right)+\left(b_{K}-b_{L}\right)\right] .
\end{aligned}
$$

Note that $\psi_{K}^{M_{T}}=0$ for all $K \in \mathcal{T}$, then a discrete integration parts yields

$$
\begin{aligned}
A_{m} & =-\sum_{n=0}^{M_{T}-1} \Delta t_{m} \sum_{K \in \mathcal{T}} m(K) \frac{\psi_{K}^{n+1}-\psi_{K}^{n}}{\Delta t_{m}} f_{K}^{n+1}-\sum_{K \in \mathcal{T}} m(K) f_{K}^{0} \psi_{K}^{0} \\
& =-\int_{0}^{T} \int_{\Omega} f_{m}(\delta \psi)_{m} \mathrm{~d} x \mathrm{~d} t-\int_{\Omega} f_{m}^{0} \psi_{m}(., 0) \mathrm{d} x,
\end{aligned}
$$

where the function $\delta \psi_{m}\left(x_{K}, t\right)=\frac{\psi_{K}^{n+1}-\psi_{K}^{n}}{\Delta t_{m}}$, if $\left(x_{K}, t\right) \in K \times\left(t^{n}, t^{n+1}\right)$. Thanks to the regularity of $\psi$, the function $\delta \psi_{m}$ converges uniformly towards $\partial_{t} \psi$ on $\Omega_{T}$. Moreover, we have

$$
f_{m} \longrightarrow f \quad \text { in } L^{2}\left(\Omega_{T}\right) \text { as } m \rightarrow \infty .
$$

Therefore

$$
\int_{0}^{T} \int_{\Omega} f_{m}(\delta \psi)_{m} \mathrm{~d} x \mathrm{~d} t \longrightarrow \int_{0}^{T} \int_{\Omega} f(x) \partial_{t} \psi \mathrm{d} x \mathrm{~d} t \quad \text { as } m \rightarrow \infty .
$$


Moreover, $f_{m}^{0}$ converges strongly in $L^{1}(\Omega)$ towards the initial data $f_{0}$ and $\psi_{m}(., 0)$ converges uniformly towards $\psi(., 0)$. Therefore, we get that

$$
\int_{\Omega} f_{m}^{0} \psi_{m}(., 0) \mathrm{d} x \longrightarrow \int_{\Omega} f_{0}(x) \psi(., 0) \mathrm{d} x \quad m \rightarrow \infty .
$$

We deduce from (36) and (37) that

$$
A_{m} \longrightarrow-\int_{0}^{T} \int_{\Omega} f(x) \partial_{t} \psi \mathrm{d} x \mathrm{~d} t-\int_{\Omega} f_{0}(x) \psi(., 0) \mathrm{d} x \quad m \rightarrow \infty .
$$

We introduce the term

$$
E_{m}=\int_{\Omega_{T}} \bar{f}_{\mathcal{D}_{m}} \nabla^{m} u_{m} \cdot \nabla \psi \mathrm{d} x \mathrm{~d} t
$$

where

$$
\bar{f}_{\mathcal{D}}(x, t)=f_{\sigma}^{n+1} \quad \forall(t, x) \in\left(t^{n}, t^{n+1}\right] \times \mathfrak{D}_{K, L}, \text { and } u=f+g+b .
$$

We have $f_{m} \underset{m \rightarrow+\infty}{\longrightarrow} f, \quad$ strongly in $L^{2}\left(\Omega_{T}\right)$. Let us to prove that

$$
\bar{f}_{\mathcal{D}_{m}}:=\bar{f}_{m} \underset{m \rightarrow+\infty}{\longrightarrow} f, \quad \text { strongly in } L^{2}\left(\Omega_{T}\right) .
$$

Since $\left\|\bar{f}_{m}-f\right\|_{L^{2}\left(\Omega_{T}\right)} \leq\left\|\bar{f}_{m}-f_{m}\right\|_{L^{2}\left(\Omega_{T}\right)}+\left\|f_{m}-f\right\|_{L^{2}\left(\Omega_{T}\right)}$, it is sufficient to prove that $\left\|\bar{f}_{m}-f_{m}\right\|_{L^{2}\left(\Omega_{T}\right)} \longrightarrow 0, \quad$ as $\delta_{m} \underset{m \rightarrow+\infty}{\longrightarrow} 0$. One has

$$
\begin{aligned}
& \left\|\bar{f}_{m}-f_{m}\right\|_{L^{2}\left(\Omega_{T}\right)}^{2}=\sum_{n=0}^{M_{T}-1} \Delta t \sum_{K \in \mathcal{T}} \sum_{\sigma \in \mathcal{E}_{\mathrm{int}, K}} m\left(\mathfrak{D}_{K, \sigma}\right)\left(f_{K}^{n+1}-f_{\sigma}^{n+1}\right)^{2} \\
& \leq \sum_{n=0}^{M_{T}-1} \Delta t \sum_{K \in \mathcal{T}} \sum_{\sigma \in \mathcal{E}_{\mathrm{int}, K}} m\left(\mathfrak{D}_{K, \sigma}\right)\left(f_{K}^{n+1}-f_{L}^{n+1}\right)^{2} \\
& \leq \sum_{n=0}^{M_{T}-1} \Delta t \sum_{\substack{\sigma \in \mathcal{E}_{\text {int }} \\
\sigma=K \mid L}} m\left(\mathfrak{D}_{K, L}\right)\left(f_{K}^{n+1}-f_{L}^{n+1}\right)^{2} \\
& \leq \frac{1}{2} \sum_{n=0}^{M_{T}-1} \Delta t \sum_{\substack{\sigma \in \mathcal{E}_{\text {int }} \\
\sigma=K \mid L}} \tau_{\sigma}\left(f_{K}^{n+1}-f_{L}^{n+1}\right)^{2} d_{\sigma}^{2} \leq \frac{C_{1}}{2} \delta_{m}^{2} .
\end{aligned}
$$

Since $\nabla^{m} u_{m}$ converges weakly in $L^{2}\left(\Omega_{T}\right)$ to $\nabla u$, since $\bar{f}_{m}$ converges strongly in $L^{2}\left(\Omega_{T}\right)$ to $f$, we have

$$
E_{m} \longrightarrow \int_{\Omega_{T}} f \nabla u \cdot \nabla \psi \mathrm{d} x \mathrm{~d} t \quad \text { as } m \rightarrow \infty .
$$

Let us to prove $E_{m}-\bar{E}_{m}$ tends to 0 as $m \rightarrow \infty$, where

$$
\bar{E}_{m}=\sum_{n=0}^{M_{T}-1} \Delta t \sum_{\substack{\sigma \in \mathcal{E}_{\mathrm{int}} \\ \sigma=K \mid L}} \tau_{\sigma} f_{\sigma}^{n+1}\left(u_{K}^{n+1}-u_{L}^{n+1}\right)\left(\psi_{K}^{n}-\psi_{L}^{n}\right) .
$$


Using the definition of discrete gradient (17), we have

$$
E_{m}=\sum_{n=0}^{M_{T}-1} \sum_{\substack{\sigma \in \mathcal{E}_{\text {int }} \\ \sigma=K \mid L}} f_{\sigma}^{n+1} \int_{t^{n}}^{t^{n+1}} \int_{\mathfrak{D}_{K, L}} \frac{m(\sigma)}{m\left(\mathfrak{D}_{K, L}\right)}\left(u_{K}^{n+1}-u_{L}^{n+1}\right) \nabla \psi \cdot \nu_{L, K} \mathrm{~d} x \mathrm{~d} t .
$$

Therefore by the definition of $\tau_{\sigma}$,

$$
\begin{aligned}
& E_{m}-\bar{E}_{m}=\sum_{n=0}^{M_{T}-1} \sum_{\substack{\sigma \in \mathcal{E}_{\text {int }} \\
\sigma=K \mid L}} m(\sigma) f_{\sigma}^{n+1}\left(u_{K}^{n+1}-u_{L}^{n+1}\right) \int_{t^{n}}^{t^{n+1}}\left(\frac{\psi_{K}-\psi_{L}}{d\left(x_{K}, x_{L}\right)}\right. \\
&\left.-\frac{1}{m\left(\mathfrak{D}_{K, L}\right)} \int_{\mathfrak{D}_{K, L}} \nabla \psi \cdot \nu_{L, K} \mathrm{~d} x\right) \mathrm{d} t .
\end{aligned}
$$

On the one hand, since the straight line $\left(x_{K}, x_{L}\right)$ is orthogonal to $\sigma$, we have $x_{K}-x_{L}=d\left(x_{K}, x_{L}\right) \nu_{L, K}$. It follows from the regularity of $\psi$ that

$$
\begin{aligned}
\frac{\psi_{K}^{n}-\psi_{L}^{n}}{d\left(x_{K}, x_{L}\right)} & =\nabla \psi\left(t^{n}, x_{L}\right) \cdot \nu_{L, K}+O(\delta) \\
& =\nabla \psi(t, x) \cdot \nu_{L, K}+O(\eta), \quad \forall(t, x) \in\left(t^{n}, t^{n+1}\right) \times \mathfrak{D}_{K, L} .
\end{aligned}
$$

By taking the mean value over $\mathfrak{D}_{K, L}$, there exists a constant $C_{5}>0$, depending only on $\psi$, such that

$$
\left|\int_{t^{n}}^{t^{n+1}}\left(\frac{\psi_{K}^{n}-\psi_{L}^{n}}{d\left(x_{K}, x_{L}\right)}-\frac{1}{m\left(\mathfrak{D}_{K, L}\right)} \int_{\mathfrak{D}_{K, L}} \nabla \psi \cdot \nu_{L, K} \mathrm{~d} x\right) \mathrm{d} t\right| \leq C_{5} \Delta t \eta .
$$

On the other hand, one has $m(\sigma)=\sqrt{\tau_{\sigma}} \sqrt{m(\sigma) d\left(x_{K}, x_{L}\right)}$. Hence by Cauchyschwarz inequality, we have

$$
\begin{aligned}
& \left|\sum_{n=0}^{M_{T}-1} \Delta t \sum_{\substack{\sigma \in \mathcal{E}_{\text {int }} \\
\sigma=K \mid L}} m(\sigma) f_{\sigma}^{n+1}\left(u_{K}^{n+1}-u_{L}^{n+1}\right)\right|^{2} \\
& \leq \sum_{n=0}^{M_{T}-1} \Delta t \sum_{\substack{\sigma \in \mathcal{E}_{\text {int }} \\
\sigma=K \mid L}} \tau_{\sigma} f_{\sigma}^{n+1}\left(u_{K}^{n+1}-u_{L}^{n+1}\right)^{2} \times \sum_{n=0}^{M_{T}-1} \Delta t \sum_{\substack{\sigma \in \mathcal{E}_{\text {int }} \\
\sigma=K \mid L}} m(\sigma) d\left(x_{K}, x_{L}\right) f_{\sigma}^{n+1} .
\end{aligned}
$$

Using Corollary 3.9 and (35) we conclude that

$$
\left|E_{m}-\bar{E}_{m}\right|^{2} \leq \frac{2 T}{\zeta}\left\|f_{0}\right\|_{L^{1}(\Omega)} C_{2} C_{5}^{2} \eta^{2} \longrightarrow 0, \quad \text { as } \eta \rightarrow 0 .
$$

This ensures that $B_{m}$ converges towards $\nu \int_{\Omega_{T}} f \nabla u \cdot \nabla \psi \mathrm{d} x \mathrm{~d} t$, as $m \rightarrow+\infty$.

\section{Numerical Results}

Let us provide some illustrations of the behavior of the numerical scheme (13)(16). The scheme leads to a nonlinear system that we solve thanks to the NewtonRaphson method. In our test case, the domain is the unit square, i.e., $\Omega=(0,1)^{2}$. We consider an admissible triangular mesh made of 14336 triangles. An illustration of a mesh type used here is given in Figure 2. The numerical analysis of the scheme was carried out for a uniform time discretization of $(0, T)$ only in order to avoid heavy notations. In order to increase the robustness of the algorithm and to ensure 
the convergence of the Newton-Raphson iterative procedure, we used an adaptive time step procedure in the practical implementation. More precisely, we associate a maximal time step $\Delta t_{\max }=0.00004$ for the mesh. If the Newton-Raphson method fails to converge after 30 iterations - we choose that the $\ell^{\infty}$ norm of the residual has to be smaller than $10^{-10}$ as stopping criterion-, the time step is divided by two. If the Newton-Raphson method converges, the time step is multiplied by two and projected on $\left[0, \Delta t_{\max }\right]$. The time step $\Delta t$ is equal to $\Delta t_{\max }$ in the test case presented below. We perform the numerical experiments with the following data

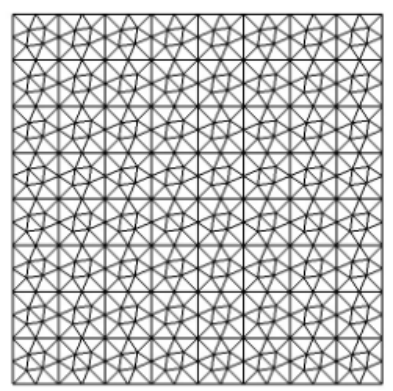

Figure 2. Mesh type used in the numerical test

$$
b(x, y)=\max \left(0, \frac{1}{2}\left(1-16(x-1 / 2)^{2}\right)((\cos (\pi y)+2)), \quad \nu=0.9 .\right.
$$

As an initial condition we take

$f_{0}(x, y)=\left\{\begin{array}{ll}\frac{1}{2} & \text { if } x \leq \frac{1}{4}, \\ 0 & \text { elsewhere, }\end{array} \quad g_{0}(x, y)= \begin{cases}b\left(\frac{1}{2}, 0\right)-b(x, y)-\left(x-\frac{1}{2}\right) & \text { if } x \leq \frac{1}{2}, \\ 0 & \text { elsewhere. }\end{cases}\right.$

Figure 3 shows the evolution of $b, \xi=b+g$ and $h=b+g+f$ at time $t=0, t=$ $0.2, t=0.72$, and $t=12$. There is convergence towards an equilibrium state, with horizontal interfaces as expected (see [19]).

Figure 4 shows the evolution of the energy along time

\section{Conclusion}

We proposed and analyzed a finite volume scheme for solving the seawater intrusion model. It preserves at the discrete level the main features of the continuous problem: the nonnegativity of the solutions, the decay of the energy, and the control of the entropy and its dissipation. Moreover, we were able to carry out a full convergence analysis based on compactness arguments.

Let us mention that to derive the problem (3), the authors in [28] assume that, for simplification, the porous medium is isotropic. Moreover in our work the mesh satisfies the so-called orthogonality condition (see, e.g., [23, Definition 9.1] so that the two-point flux approximation is consistent. We know that the finite volume method with two-point flux approximation, used here, does not allow to handle the anisotropic case. Nevertheless in order to treat this case, we can use for instance a Control Volume Finite Element scheme (CVFE) [27, 11, 2]. 

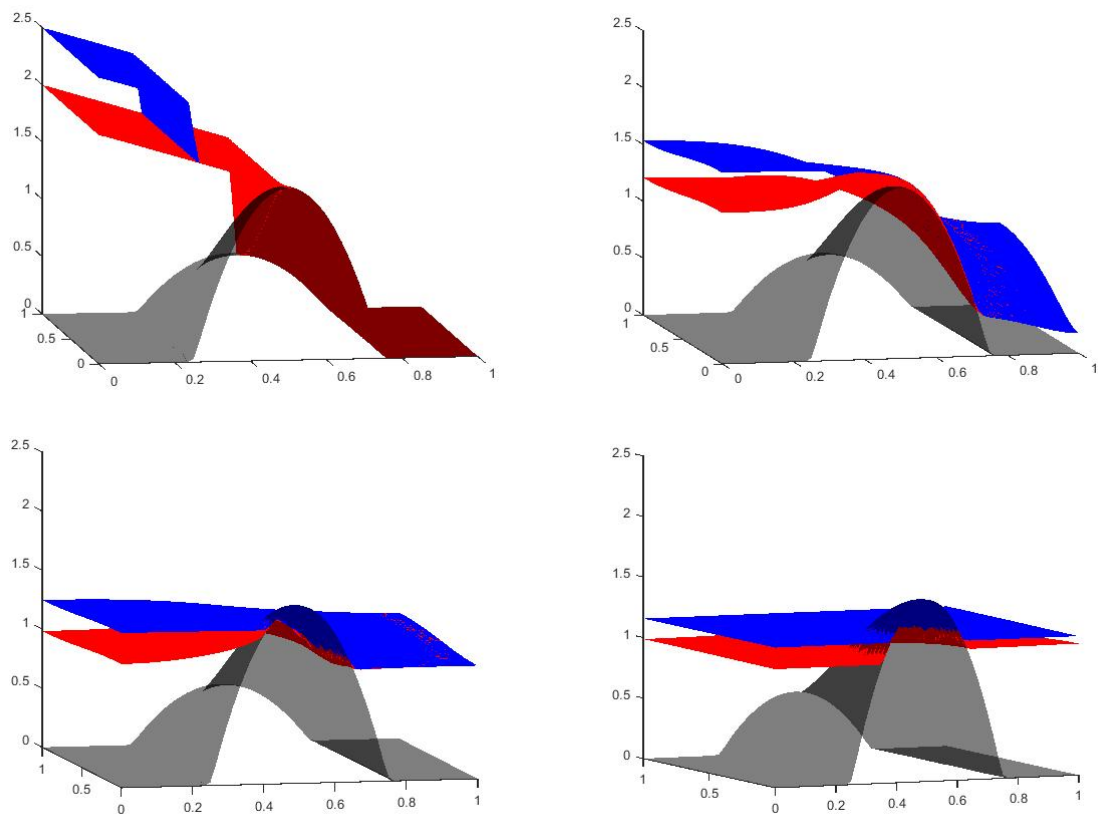

Figure 3. Behaviour of the model at $t=0, t=0.2, t=0.72, t=12$

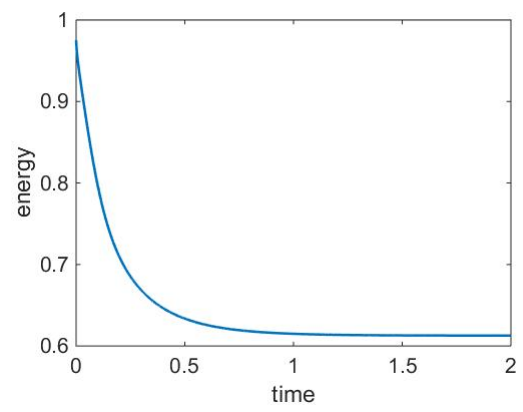

FIgURE 4. Evolution of the energy along time

\section{ACKNOWLEDGEMENTS}

The author thanks the team Inria/Rapsodi, the Labex CEMPI (ANR-11-LABX0007-01), and the project GEOPOR (ANR-13-JS01-0007-01) for their support. The author also thanks Claire Chainais-Hillairet and Clément Cancès for fruitful discussions.

\section{REFERENCES}

[1] A. Abudawia and C. Rosier. Numerical analysis for a seawater intrusion problem in a confined aquifer. Math. Comput. Simulation, 118:2-16, 2015. 
[2] A. Ait Hammou Oulhaj, C. Cancès, and C. Chainais-Hillairet. Numerical analysis of a nonlinearly stable and positive control volume finite element scheme for Richards equation with anisotropy. HAL: hal-01372954, 2016.

[3] A. Al Bitar. Modélisation des écoulements en milieu poreux hétérogenes 2D/3D, avec couplages surface/souterrain et densitaires. PhD thesis, Institut National Polytechnique de Toulouse, 2007.

[4] H. W. Alt and S. Luckhaus. Quasilinear elliptic-parabolic differential equations. Math. Z., 183(3):311-341, 1983.

[5] B. Andreianov, M. Bendahmane, and R. Ruiz-Baier. Analysis of a finite volume method for a cross-diffusion model in population dynamics. Math. Models Methods Appl. Sci., 21(02):307$344,2011$.

[6] B. Andreianov, C. Cancès, and A. Moussa. A nonlinear time compactness result and applications to discretization of degenerate parabolic-elliptic pdes. HAL: hal-01142499, 2015.

[7] J. Bear. Dynamic of fuilds in Porous media. American Elsevier, New York, 1972.

[8] J. Bear. Hydraulics of groundwater. McGraw-Hill series in water resources and environmental engineering. McGraw-Hill International Book Co., 1979.

[9] J. Bear, A. H.-D. Cheng, S. Sorek, D. Ouazar, and I. Herrera. Seawater intrusion in coastal aquifers: concepts, methods and practices, volume 14. Springer Science \& Business Media, 1999.

[10] M. Bessemoulin-Chatard and A. Jüngel. A finite volume scheme for a Keller-Segel model with additional cross-diffusion. IMA J. Numer. Anal., 34(1):96-122, 2014.

[11] C. Cancès and C. Guichard. Convergence of a nonlinear entropy diminishing control volume finite element scheme for solving anisotropic degenerate parabolic equations. Math. Comp., $85(298): 549-580,2016$.

[12] C. Chainais-Hillairet, J.-G. Liu, and Y.-J. Peng. Finite volume scheme for multi-dimensional drift-diffusion equations and convergence analysis. M2AN Math. Model. Numer. Anal., $37(2): 319-338,2003$.

[13] Z. Chen and R. Ewing. Mathematical analysis for reservoir models. SIAM J. Math. Anal., 30(2):431-453, 1999.

[14] C. Choquet. Parabolic and degenerate parabolic models for pressure-driven transport problems. Math. Models Methods Appl. Sci., 20(4):543-566, 2010.

[15] C. Choquet, M. M. Diédhiou, and C. Rosier. Mathematical analysis of a sharp-diffuse interfaces model for seawater intrusion. J. Differential Equations, 259(8):3803-3824, 2015.

[16] C. Choquet, M. M. Diédhiou, and C. Rosier. Derivation of a sharp-diffuse interfaces model for seawater intrusion in a free aquifer. numerical simulations. SIAM J. Appl. Math., 76(1):138$158,2016$.

[17] C. Choquet, J. Li, and C. Rosier. Global existence for seawater intrusion models: comparison between sharp interface and sharp-diffuse interface approaches. Electron. J. Differential Equations, pages No. 126, 27, 2015.

[18] K. Deimling. Nonlinear functional analysis. Springer-Verlag, Berlin, 1985.

[19] J. Escher, P. Laurençot, and B.-V. Matioc. Existence and stability of weak solutions for a degenerate parabolic system modelling two-phase flows in porous media. Ann. Inst. H. Poincaré Anal. Non Linéaire., 28(4):583-598, 2011.

[20] J. Escher, A.-V. Matioc, and B.-V. Matioc. Modelling and analysis of the Muskat problem for thin fluid layers. J. Math. Fluid Mech., 14(2):267-277, 2012.

[21] J. Escher and B.-V. Matioc. Existence and stability of solutions for a strongly coupled system modelling thin fluid films. NoDEA Nonlinear Differential Equations Appl., 20(3):539-555, 2013.

[22] R. Eymard, T. Gallouët, M. Ghilani, and R. Herbin. Error estimates for the approximate solutions of a nonlinear hyperbolic equation given by finite volume schemes. IMA J. Numer. Anal., 18(4):563-594, 1998.

[23] R. Eymard, T. Gallouët, and R. Herbin. Finite volume methods. In Handbook of numerical analysis, Vol. VII, Handb. Numer. Anal., VII, pages 713-1020. North-Holland, Amsterdam, 2000.

[24] R. Eymard and T. Gallouët. $H$-convergence and numerical schemes for elliptic problems. SIAM J. Numer. Anal., 41(2):539-562, 2003.

[25] R. Eymard, R. Herbin, and A. Michel. Mathematical study of a petroleum-engineering scheme. M2AN Math. Model. Numer. Anal., 37(6):937-972, 2003. 
[26] F. Filbet. A finite volume scheme for the Patlak-Keller-Segel chemotaxis model. Numer. Math., 104(4):457-488, 2006.

[27] P. A. Forsyth. A control volume finite element approach to NAPL groundwater contamination. SIAM J. Sci. Statist. Comput., 12(5):1029-1057, 1991.

[28] M. Jazar and R. Monneau. Derivation of seawater intrusion models by formal asymptotics. SIAM J. Appl. Math., 74(4):1152-1173, 2014.

[29] P. Laurençot and B.-V. Matioc. A gradient flow approach to a thin film approximation of the Muskat problem. Calc. Var. Partial Differential Equations, 47(1-2):319-341, 2013.

[30] P. Laurençot and B.-V. Matioc. A thin film approximation of the Muskat problem with gravity and capillary forces. J. Math. Soc. Japan, 66(4):1043-1071, 2014.

[31] J. Leray and J. Schauder. Topologie et équations fonctionnelles. Ann. Sci. École Norm. Sup. (3)., 51:45-78, 1934.

[32] P. Marion, K. Najib, and C. Rosier. Numerical simulations for a seawater intrusion problem in a free aquifer. Appl. Numer. Math., 75:48-60, 2014.

[33] A. Michel. A mathematical comparison of two finite volume methods for two phase flow in porous media. In Finite volumes for complex applications, III (Porquerolles, 2002), pages 213-220. Hermes Sci. Publ., Paris, 2002.

[34] K. Najib and C. Rosier. On the global existence for a degenerate elliptic-parabolic seawater intrusion problem. Math. Comput. Simulation, 81(10):2282-2295, 2011.

[35] O. Strack, R. Barnes, and A. Verruijt. Vertically integrated flows, discharge potential, and the Dupuit-Forchheimer approximation. Ground water, 44(1):72-75, 2006.

[36] O. D. Strack. A Dupuit-Forchheimer model for three-dimensional flow with variable density. Water Resources Research, 31(12):3007-3017, 1995.

[37] M. E. A. Talibi and M. H. Tber. Existence of solutions for a degenerate seawater intrusion problem. Electron. J. Differential Equations, 2005(72):1-14, 2005.

[38] M. H. Tber, M. E. A. Talibi, and D. Ouazar. Parameters identification in a seawater intrusion model using adjoint sensitive method. Math. Comput. Simulation, 77(2):301-312, 2008.

Ahmed Ait Hammou Oulhaj

Laboratoire Paul Painlevé, UMR CNRS 8524

Université Lille 1

59655 Villeneuve d'Ascq Cedex

ahmed.ait-hammou-oulhaj@math.univ-lille1.fr 